\title{
Magnetic Properties of the Nanocrystalline $\mathrm{DyMnO}_{3}$ Compound
}

\author{
V. Dyakonov ${ }^{a, b}$, A. Szytula ${ }^{c}$, S. Baran $^{c}$, Z. Kravchenko $^{b}, \mathrm{E} \mathrm{Zubov}^{b}$,

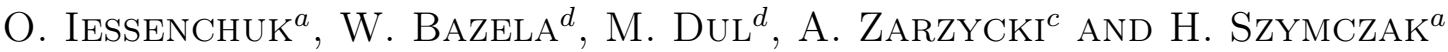 \\ ${ }^{a}$ Institute of Physics, PAS, al. Lotników 32/46, 02-668 Warsaw, Poland \\ ${ }^{b}$ A.A. Galkin Donetsk Physico-Technical Institute, NANU, R. Luxembourg St. 72, 83114 Donetsk, Ukraine \\ ${ }^{c}$ M. Smoluchowski Institute of Physics, Jagiellonian University, Reymonta 4, 30-059, Kraków, Poland \\ ${ }^{d}$ Institute of Physics, Technical University, Podchorążych 1, 30-084 Kraków, Poland
}

\begin{abstract}
We report on the X-ray powder diffraction and magnetic measurements of the polycrystalline and the two nanocrystalline samples of $\mathrm{DyMnO}_{3}$ compound synthesized at temperatures of 800 and $850{ }^{\circ} \mathrm{C}$. All samples at room temperature crystallize in the orthorhombic crystal structure (space group Pnma). The crystal structure parameters determined change only slightly with preparation methods but the average grains size of the nanoparticles determined from X-ray data increases significantly with increasing annealing temperature. Temperature dependence of the magnetic susceptibility indicates the antiferromagnetic order in Dy sublattice at low temperatures.
\end{abstract}

PACS numbers: 61.05.cp, 75.47.Lx, 75.50.Ee, 75.75.-c

\section{Introduction}

Physical properties of manganites were shown to depend on doping as well as on the grains size $[1,2]$. Usually, the nanocrystalline samples exhibit properties which are different from those of the bulk material. An important factor is increasing surface-to-volume ratio of the grains as the particle size is reduced to the nanoscale. As a result, the small size effect and the surface effect perturb the properties of nanoparticle samples. In these samples the magnetic behaviour of nanoparticles is additionally modified by the interparticle interactions which can be both of the exchange and magnetostatic origins. The main motivation for these studies was to obtain the data concerning the evolution of magnetic properties as a function of grain size.

$\mathrm{RMnO}_{3}$ compounds have been attracting a lot of attention in the last years due to strong coupling between ferroelectricity and magnetism [3]. These compounds crystallize in the orthorhombic crystal structure (space group Pnma).

In $\mathrm{DyMnO}_{3}$ below $6.5 \mathrm{~K}$, the Dy magnetic moments order in a commensurate structure with the propagation vector $\boldsymbol{k}=(0,1 / 2,0)$, while the Mn moments order below $39 \mathrm{~K}$. At $T_{\mathrm{t}}=18 \mathrm{~K}$ the change of the magnetic structure connected with the Mn sublattice is observed. Below $T_{\mathrm{t}}$ this order is collinear while above $T_{\mathrm{t}}$ it is sine modulated [4].

This work reports the results of the X-ray diffraction and magnetometric macroscopic measurements of the $\mathrm{DyMnO}_{3}$ polycrystalline and nanocrystalline samples with the different average grain size.

\section{Experimental and results}

The polycrystalline manganite $\mathrm{DyMnO}_{3}$ (denoted hereafter as $S_{1}$ sample) was prepared by the solid-state reaction. The final sintering treatment was performed at $1150{ }^{\circ} \mathrm{C}$ for $15 \mathrm{~h}$.

For the preparation of nanosize manganite $\mathrm{DyMnO}_{3}$ the sol-gel method has been used. The mixture of stoichiometric amounts of high purity $\mathrm{Mn}_{3} \mathrm{O}_{4}$ and $\mathrm{Dy}_{2} \mathrm{O}_{3}$ oxides was dissolved in acetic acid with added hydrogen peroxide. The solution of urea was added to the obtained solution. This mixture was evaporated to dryness. The dry remainder was decomposed at $250-450{ }^{\circ} \mathrm{C}$. Then, the powder was thoroughly grinded into pellets and was annealed at temperatures of 800 and $850^{\circ} \mathrm{C}$ for $20 \mathrm{~h}$ in air, followed by a slow cooling down to the room temperature. The samples of the nanopowders obtained after annealing at 800 and $850{ }^{\circ} \mathrm{C}$ are denoted hereafter as $S_{2}$ and $S_{3}$, respectively.

The quality of the products was checked by X-ray powder diffraction at room temperature using a Philips PW-3710 X'PERT diffractometer with $\mathrm{Cu} K_{\alpha}$ radiation. The obtained data were analyzed with the Rietveld-type refinement software FullProf program [5]. For all samples the following dc magnetic measurements of nanosize samples using a commercial MPMS SQUID magnetometer have been carried out: magnetic susceptibility measurements in a magnetic field of $1 \mathrm{kOe}$ over a temperature 
range $2-300 \mathrm{~K}$ (from these data the effective magnetic moment $\mu_{\text {eff }}$ and the paramagnetic Curie temperature $\theta_{\mathrm{p}}$ were obtained) and magnetization measurements in magnetic fields up to $50 \mathrm{kOe}$ and at temperatures down to $2 \mathrm{~K}$ (in order to get the value of the pseudosaturated magnetic moment and character of the magnetization curve).

Additional magnetization vs. temperature measurements of the polycrystalline sample $S_{1}$ in the temperature range of $4.2-120 \mathrm{~K}$ and in the external field from 50 Oe to $5 \mathrm{kOe}$ and the external field up to $15 \mathrm{kOe}$ were performed using PAR Model 4500 vibrating sample magnetometer.

The X-ray diffraction data indicate that all the samples studied have orthorhombic crystal structure described by the space group Pnma. In this structure the respective atoms occupy the following atoms: $\mathrm{R}$ and $\mathrm{O} 1$ in $4 \mathrm{c}$ site: $(x, y, 1 / 4), \mathrm{O} 2$ in 81 site: $(x, y, z)$ and $\mathrm{Mn}$ in $4 \mathrm{~b}$ site: $(1 / 2,0,0)$. All fitted structural parameters: lattice constants and positional parameters $x_{i}, y_{i}$ and $z_{i}$ are listed in Table I. The obtained data indicate that the crystal structure parameters slightly change with the nanostructuration of the samples.

TABLE I

Structural parameters of $\mathrm{DyMnO}_{3}$ at room temperature derived from X-ray diffraction data $\left(S_{1}\right.$ - polycrystalline sample, $S_{2}$ and $S_{3}$ - the nanocrystalline samples annealed at 800 and $850{ }^{\circ} \mathrm{C}$, respectively).

\begin{tabular}{c|c|c|c}
\hline \hline Samples & $S_{1}$ & $S_{2}$ & $S_{3}$ \\
\hline $\mathrm{a}[\AA]$ & $5.8385(2)$ & $5.8334(1)$ & $5.8306(1)$ \\
$\mathrm{b}[\AA]$ & $7.3792(2)$ & $7.3794(2)$ & $7.3806(1)$ \\
$\mathrm{c}[\AA]$ & $5.2789(1)$ & $5.2742(1)$ & $5.2738(1)$ \\
$\mathrm{V}\left[\AA^{3}\right]$ & $227.433(19)$ & $227.04(1)$ & $226.95(1)$ \\
$\mathrm{R} x$ & $0.0819(2)$ & $0.0820(1)$ & $0.0822(1)$ \\
$y$ & $-0.0139(4)$ & $-0.0156(2)$ & $-0.0185(2)$ \\
$\mathrm{O} 1 x$ & $0.473(2)$ & $0.462(2)$ & $0.463(1)$ \\
$y$ & $0.102(3)$ & $0.104(2)$ & $0.105(1)$ \\
$\mathrm{O} 2 x$ & $0.324(2)$ & $0.327(1)$ & $0.323(1)$ \\
$y$ & $0.049(1)$ & $0.054(1)$ & $0.052(1)$ \\
$z$ & $0.705(2)$ & $0.699(1)$ & $0.704(1)$ \\
$R_{\text {Bragg }}[\%]$ & 8.1 & 6.6 & 6.6 \\
$R_{\text {prof }}$ & 10.0 & 9.3 & 6.3
\end{tabular}

In the next step, the grain sizes were determined using the Scherrer relation $d=(\lambda / B) \cos \theta_{\mathrm{B}}$, where $d$ is the grain size, $\lambda=0.154178 \mathrm{~nm}$ - the $\mathrm{X}$-ray wavelength, $\theta_{\mathrm{B}}$ - the corresponding angle of Bragg diffraction and $B$ the difference between half-widths of the Bragg reflex of the nanopowder and the standard sample ( $\mathrm{Si}$ powder with the size $10 \mu \mathrm{m}[6])$. The grains sizes were calculated using the experimental X-ray data and the following three relations:

1. $B_{1}=\beta-\beta_{0}$, where $\beta$ is the half-widths of the Bragg reflex of the investigated sample and $\beta_{0}$ the similar value for the standard sample of Si powder,

$$
\begin{aligned}
& \text { 2. } B_{2}=\sqrt{\beta^{2}-\beta_{0}^{2}}, \\
& \text { 3. } B_{3}=\sqrt{\left(\beta-\beta_{0}\right) \sqrt{\beta^{2}-\beta_{0}^{2}}} \text {. }
\end{aligned}
$$

The grain size values $d_{i}(i=1,2,3)$ determined from these relations are listed in Table II. Presented data indicate that the grain size increases with increasing annealing temperature.

TABLE II

Particle size values, $d\left(S_{i}\right)$ (in $\mathrm{nm}$ ), of $\mathrm{DyMnO}_{3}$ nanopowder prepared at $800{ }^{\circ} \mathrm{C}\left(S_{2}\right)$ and $850{ }^{\circ} \mathrm{C}\left(S_{3}\right)$ calculated by the different methods (see text).

\begin{tabular}{c|c|c|c}
\hline \hline $\mathrm{DyMnO}_{3}$ & $B_{1}$ & $B_{2}$ & $B_{3}$ \\
\hline$d\left(S_{2}\right)$ & 31.6 & 25.2 & 28.2 \\
$d\left(S_{3}\right)$ & 57.2 & 40.0 & 47.8
\end{tabular}

Temperature dependences of magnetization for the polycrystalline sample $S_{1}$ measured at different external magnetic field are shown in Fig. 1. The $M(T)$ maximum at $T=8 \mathrm{~K}$, which is near the reported value $T_{\mathrm{N}}(D y)=6.5 \mathrm{~K}[5]$, indicates the antiferromagnetic order of the Dy moments. The $M(T)$ maximum temperature is seen to be not dependent on magnetic field up to $5 \mathrm{kOe}$.

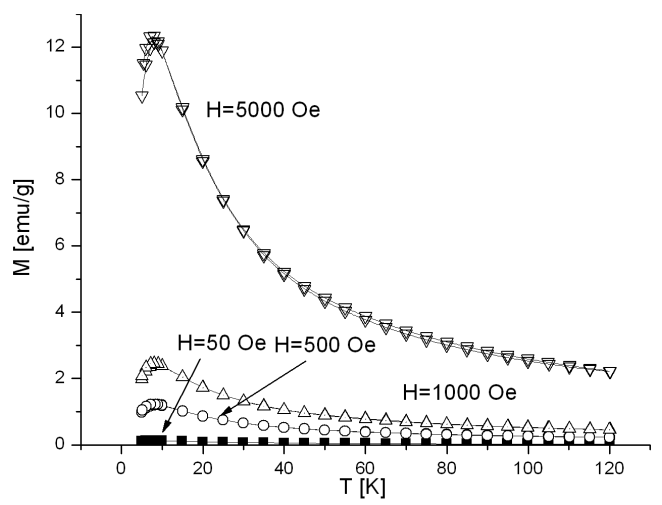

Fig. 1. Temperature dependences of magnetization for the polycrystalline sample $\mathrm{DyMnO}_{3}\left(S_{1}\right)$ measured at different magnetic fields.

The temperature dependences of reciprocal magnetic susceptibility and dc magnetic susceptibility for three samples $S_{1}, S_{2}$ and $S_{3}$ at low temperatures are shown in Fig. 2. Above $50 \mathrm{~K}$, the reciprocal magnetic susceptibility follows the Curie-Weiss law with the negative values of the paramagnetic Curie temperature $\theta_{\mathrm{p}}$ and the effective magnetic moment equal 11.8, 11.3 and $11.5 \mu_{\mathrm{eff}}$ for $S_{1}, S_{2}$ and $S_{3}$ samples, respectively, and larger than that of the free $\mathrm{Dy}^{3+}$ ion value $\left(10.65 \mu_{\mathrm{B}}\right)$ (see Table III). This indicates that also on the Mn atoms is localized magnetic moment. The $\operatorname{sign} \theta_{\mathrm{p}}$ indicates that antiferromagnetic interactions between the magnetic moments are dominant.

The temperature dependence of the magnetic susceptibility for the all samples (see inset in Fig. 2) shows 


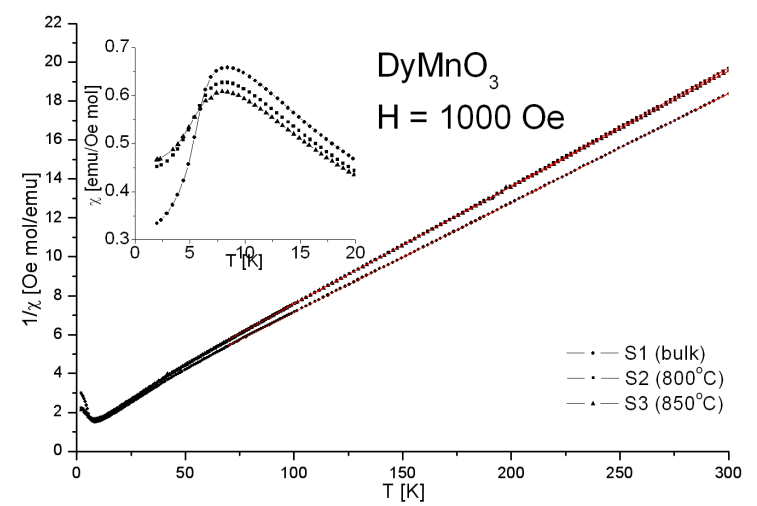

Fig. 2. Temperature dependences of the reciprocal magnetic susceptibility of polycrystalline and nanosized $\mathrm{DyMnO}_{3}$ samples. Inset shows the low temperature part of magnetic susceptibility. The data for $S_{2}(\boldsymbol{\square})$ and $S_{3}(\boldsymbol{\Lambda})$ coincide in the large temperature region.

TABLE III

Magnetic data for $\mathrm{DyMnO}_{3}\left(S_{1}\right.$ - polycrystalline sample, $S_{2}$ and $S_{3}$ - samples annealed at 800 and $850{ }^{\circ} \mathrm{C}$, respectively), $T_{\mathrm{N}}$, (Dy) - Nèel temperature, $\theta_{\mathrm{p}}$ - paramagnetic Curie temperature, $\mu_{\mathrm{eff}}-$ effective magnetic moment in paramagnetic state, $\mu$ - magnetic moment at $T=2 \mathrm{~K}$ and magnetic field $H=50 \mathrm{kOe}$.

\begin{tabular}{c|c|c|c|c}
\hline \hline Sample & $T_{\mathrm{N}, \mathrm{Dy}}[\mathrm{K}]$ & $\theta_{\mathrm{p}}[\mathrm{K}]$ & $\mu_{\text {eff }}\left[\mu_{\mathrm{B}}\right]$ & $\mu\left[\mu_{\mathrm{B}}\right]$ \\
\hline$S_{1}$ & 8.5 & -26.6 & 11.8 & 4.8 \\
$S_{2}$ & 8.1 & -24.8 & 11.3 & 4.4 \\
$S_{3}$ & 8.3 & -24.0 & 11.5 & 4.5
\end{tabular}

maximum at temperature of for example $T_{\mathrm{N}}^{\mathrm{Dy}}=8.5 \mathrm{~K}$ for $S_{1}$ corresponding to the antiferromagnetic ordering of the Dy moments. Similar values are observed for the $S_{2}$ and $S_{3}$ samples (see Table III).

Figure 3 shows the magnetization vs. field curves for the $S_{1}$ sample measured at temperatures of 5,42 and $100 \mathrm{~K}$ in the external fields up to $15 \mathrm{kOe}$ for the increase

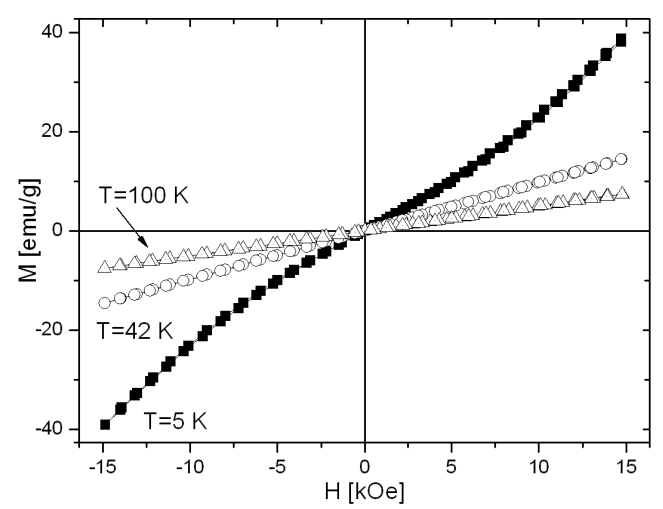

Fig. 3. Magnetization curves for the polycrystalline sample $\mathrm{DyMnO}_{3}\left(S_{1}\right)$ measured in the field of $\pm 15 \mathrm{kOe}$ at $T=5,42$ and $100 \mathrm{~K}$.

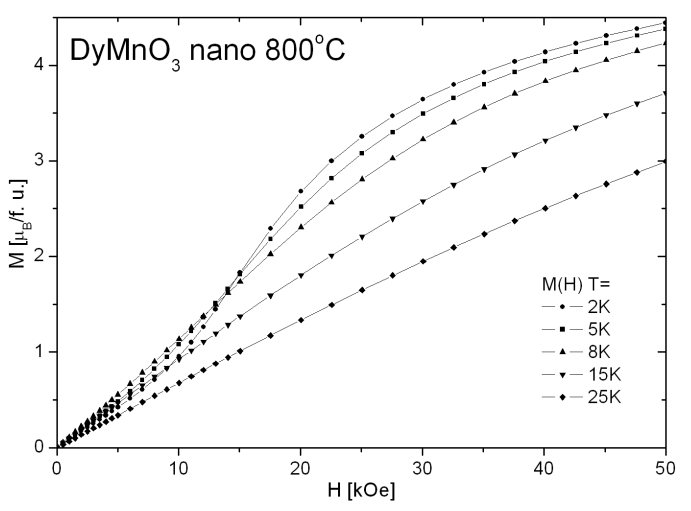

Fig. 4. Magnetization vs external magnetic field for the nanosized $\mathrm{DyMnO}_{3}\left(S_{2}\right.$ sample $)$ at $2,5,8.0,15$ and $25 \mathrm{~K}$.

and decrease of the magnetic field. These dependence are linear for $T=42$ and $100 \mathrm{~K}$. For $T=5 \mathrm{~K}$ a deviation from linear dependence above $H=5 \mathrm{~K}$ is observed. All curves do not exhibit the hysteresis.

In Fig. 4, the magnetization isotherm for the $S_{2}$ sample measured at $T=2,5,8,15$ and $25 \mathrm{~K}$ in the magnetic field up to $50 \mathrm{kOe}$ is present. The $M(H)$ curve at $2 \mathrm{~K}$ shows the magnetic field-induced magnetic transition in the rare earth sublattice near $5 \mathrm{kOe}$, thus proving an essentially antiferromagnetic nature of the ground state. The magnetization curves are not saturated in the magnetic field equal to $50 \mathrm{kOe}$. The magnetic moment value at $T=2 \mathrm{~K}$ and $H=50 \mathrm{kOe}$ are smaller than that for free $\mathrm{Dy}^{3+}$ ion value $\left(10 \mu_{\mathrm{B}}\right)$ (see Table III).

\section{Conclusions}

In this paper, the results of the X-ray diffraction and magnetometric measurements of the $\mathrm{DyMnO}_{3}$ prepared by the various methods are presented. All investigated samples have the orthorhombic crystal structure described by the space group Pnma. The crystal structure parameters change insignificantly with the preparation method. The grains size increases with increasing annealing temperature.

Magnetic data indicate that all the samples are antiferromagnets. The $M(T)$ dependences presented clearly show the anomalies at low temperatures corresponding to the Dy magnetic moment ordering. The values of effective magnetic moment suggest the localization of the magnetic moment on the dysprosium and manganese atoms. The values of the magnetic parameters: the Nèel and paramagnetic Curie temperatures and effective magnetic field magnetic moments do not change with the magnitude of the grains size.

\section{Acknowledgments}

This work was financially supported by European Fund for Regional Development (contract No. UDA-POIG.01.03.01-00-058/08/00). 


\section{References}

[1] L.I. Balcells, J. Fontcuberta, B. Martinez, X. Obradors, Phys. Rev. B 58 R14697 (1998).

[2] Y.W. Duan, X.L. Kon, J.G. Li, Physica B, Condens. Matter 355, 250 (2005).

[3] M. Fiebig, J. Phys. D 38 R 123 (2005).
[4] R. Feyerherm, E. Dudzik, N. Aliouane, D.N. Argyriou, Phys. Rev. B 73 R180401 (2006).

[5] J. Rodriguez-Carvajal, Physica B 192, 55 (1993).

[6] S.D. Rasberry, Bureau of Standards Certificate-Standard Reference Material 640b, 1987.

[7] T. Goto, T. Kimura, G. Lawet, A.P. Ramirez, Y. Tokura, Phys. Rev. Lett. 92, 257201 (2004). 